\title{
GeometriAR: aplicativo educacional com realidade aumentada para auxiliar o ensino de sólidos geométricos
}

\author{
Allisson Pierre Lino Gomes, UNIVASF, Brasil, allissonpierre@ outlook.com \\ Ricardo Argenton Ramos, UNIVASF, Brasil, ricargentonramos@gmail.com \\ Lucas Florêncio de Brito, UNIVASF, Brasil, lucasdibex@gmail.com \\ Michel Ferreira Batista, UNIVASF, Brasil, michelfbatista@ hotmail.com \\ Brauliro Gonçalves Leal, UNIVASF, Brasil, brauliro.leal@gmail.com
}

\begin{abstract}
Resumo: Pesquisas mostram que o ensino de matemática tem baixa eficiência no Brasil. Uma possível solução para ajudar na melhoria do ensino é a utilização de recursos computacionais. Entre esses recursos, a realidade aumentada (RA) vem ganhando destaque por facilitar a percepção de objetos em 3D. Assim, este artigo apresenta o GeometriAR, um aplicativo educacional que utiliza RA para contribuir no ensino de geometria espacial. O aplicativo reproduz sólidos geométricos em 3D a partir de imagens de figuras planas. Para validação do aplicativo foi feita uma pesquisa com professores de matemática, considerando aspectos gerais, pedagógicos, de usabilidade, de conteúdo e interface. Com os resultados obtidos, concluiu-se que o aplicativo pode ser utilizado como ferramenta auxiliar no ensino de geometria espacial em sala de aula.
\end{abstract}

Palavras-chave: Matemática. Realidade Aumentada. Aplicativo Educacional. Geometria Espacial.

\section{GeometriAR: educational app with augmented reality to aid the teaching of geometric solids}

\begin{abstract}
Research shows that mathematics teaching has low efficiency in Brazil. Among the resources, augmented reality (AR) has been gaining prominence by arousing interest in students. Thus, this article presents the GeometriAR, an educational application that uses RA to contribute in the teaching of spatial geometry. The application plays $3 D$ geometric solids from flat figures images. To validate the application, a research was done with mathematics teachers, considering general, pedagogical, usability, content and interface aspects. With the obtained results, it was concluded that the application can be used as an auxiliary tool in the teaching of spatial geometry in the classroom.
\end{abstract}

Keywords: Mathematics. Augmented Reality. Education app. Spatial Geometry.

\section{Introdução}

De acordo com os resultados do Sistema de Avaliação da Educação Básica (SAEB) de 2017, apresentados pelo Instituto Nacional de Estudos e Pesquisas Educacionais Anísio Teixeira (Inep, 2018), somente 4,5\% dos estudantes brasileiros da $3^{a}$ série do ensino médio têm o nível de proficiência considerado adequado em matemática. Essa avaliação revelou que $71,7 \%$ dos estudantes apresentaram nível de proficiência em matemática insuficiente e 23,8\% apresentaram o nível básico.

Para Marques et al. (2018), as dificuldades no ensino em matemática podem ser impactadas por vários motivos, dentre eles estão as metodologias adotadas pelos docentes, em que os alunos as consideram desinteressantes ou até mesmo ultrapassadas, e ainda indicam que o conteúdo da disciplina é muito difícil, sendo essa combinação prejudicial ao aprendizado. Assim, percebe-se que diversificar metodologias de ensino pode ser interessante para estimular o aluno e facilitar na compreensão de conteúdos. 
Dentro do contexto de ensino e aprendizagem da geometria espacial no Brasil, a análise dos resultados do Programme for International Student Assessment (PISA) de 2015, divulgada pelo Inep (2016), revelou que dentre 4 categorias de matemática, os alunos de 15 anos do Brasil têm mais dificuldade na categoria de "espaço e forma", em que pode-se trabalhar com propriedades de figuras geométricas, de figuras espaciais, entre outras. Nessa avaliação do PISA, determinou-se que a interação dinâmica com formas reais e suas representações era o conteúdo mais difícil e trabalhoso.

Chaves (2013) observou que problemas em geometria espacial podem se iniciar com a dificuldade em aprendizagem de geometria plana e se intensificar ao trabalhar com áreas, volumes e planificações de objetos tridimensionais (3D). Vidaletti (2009, p. 15) atribui algumas dificuldades em geometria espacial a uma "falta de informação e de construção do conhecimento de forma gradual, concreta, interessante e significativa para os alunos". Para sanar alguns problemas, trabalhos recentes (Salin, 2013; Volpatto et al., 2018) propõem atividades em sala de aula que trazem a visualização de figuras espaciais, seja por meio de materiais manipuláveis físicos ou digitais.

Dentre as alternativas para diversificar metodologias de ensino, pode ser notado o uso das tecnologias, como é dito no artigo de Menegai et al. (2018). Nele é descrito que a utilização desse tipo de recurso tem seus benefícios no contexto educacional, porém os educadores devem se aprimorar para lidar de forma correta com as tecnologias a serem adotadas, a fim de que seja possível a construção do conhecimento de forma adequada em matemática. Com isso, nota-se que novas tecnologias ou softwares educacionais podem levar a importantes contribuições para a educação.

Para Akçayir e Akçayir (2017), a realidade aumentada (RA) é uma tecnologia em crescimento que possibilita a união do ambiente virtual com o real, permitindo, assim, que o usuário aumente sua percepção, como é o caso para objetos em três dimensões. Eles e outros autores como Masmuzidin e Aziz (2018) também apontam que vem crescendo a tendência do uso de RA para o ensino de ciências, tecnologias, engenharias e matemática, pois concluem que este recurso torna as aulas mais dinâmicas do que as tradicionais, além de trazer um maior poder de atração e consequentemente uma melhor compreensão do conteúdo.

Mediante o contexto até aqui apresentado, este artigo é guiado pela seguinte questão: do ponto de vista de professores de matemática, aplicativos com RA podem ser aplicados em sala de aula para auxiliar no ensino de sólidos geométricos? Considerando-se nesta avaliação aspectos gerais, pedagógicos, de usabilidade, conteúdo e interface. Portanto, o objetivo deste artigo é apresentar o aplicativo GeometriAR, um aplicativo educacional com RA para auxiliar no ensino de sólidos geométricos, e a sua avaliação do ponto de vista de especialistas na área de ensino de matemática.

Este artigo está organizado da seguinte forma: após esta introdução é conceituada a realidade aumentada e seu potencial na área da educação. Em seguida, são apresentados os materiais e métodos utilizados na construção e avaliação do aplicativo. Na sequência, são apresentados os resultados e as discussões sobre o aplicativo, concluindo com as considerações finais e referências utilizadas neste estudo.

\section{A Realidade Aumentada e seu potencial para o auxílio educacional}

A Realidade Aumentada não é um conceito novo, desde a década de 90 os pesquisadores Milgram et al. (1995) definiram a RA como uma realidade mista, em que objetos reais e virtuais coexistem em uma única tela, sendo que o utilizador tem a visão composta do real com o virtual. Na mesma década, para evitar a limitação do conceito da RA atrelada a determinadas tecnologias Azuma (1997) propôs que a realidade 
aumentada fosse definida a partir de sistemas que apresentam três características: combinação entre real e virtual, interativo em tempo real e registrado em 3D.

Dado a definição, Cheng e Tsai (2013) destacam que a RA pode projetar imagens em 3D através de duas maneiras: com base em uma figura (um marcador), ou através da localização utilizando uma rede sem fio ou sistema de posicionamento global (GPS). Cadavieco et al. (2014) pontuam que essa relação entre objetos físicos e virtuais da RA pode ser um complemento envolvente e útil para educação, sendo também que a interação com objetos em 3D permite aos alunos uma melhor percepção da realidade e pode estimulá-los para uma maior capacidade de raciocínio abstrato e espacial.

Existem diversos campos em que a RA pode ser aplicada. No âmbito educacional, Mekni e Lemieux (2014) relatam que novas possibilidades de ensino e aprendizagem fornecidas por RA têm sido cada vez mais reconhecidas por pesquisadores educacionais. O trabalho de Herpich et al. (2018) é um exemplo que traz resultados favoráveis para RA na educação, nele foi verificado ganho de atenção dos estudantes brasileiros durante uma atividade de estudo de física utilizando RA (com o software avatAR), quando comparado o estudo utilizando o software Moodle.

Percebendo-se algumas tendências da RA para a área da Educação, Akçayir e Akçayir (2017) fizeram uma revisão sistemática e agruparam 68 artigos científicos que apresentam as vantagens de utilizar a tecnologia de RA para o ensino em conjunto com alguma técnica ou em comparação com outras técnicas de ensino. Ainda nesse trabalho, os autores pontuam alguns desafios para a área, entre eles: o valor dos equipamentos, a falta de contextualização dos softwares com o cenário da escola e, ainda, a falta de pesquisas para avaliar efetivamente as vantagens na perspectiva educacional. $\mathrm{O}$ desenvolvimento do aplicativo GeometriAR tem como base esses desafios.

\section{Materiais e métodos}

A metodologia utilizada no presente artigo foi dividida em duas partes. A primeira utilizou as boas práticas da engenharia de software e o foco em atender as funcionalidade do ponto de vista educacional. Na segunda parte o foco foi avaliar o aplicativo do ponto de vista de professores da área de Matemática.

Parte 1: Para atender parte dos desafios pautados em Akçayir e Akçayir (2017) foi definido que o aplicativo iria funcionar em dispositivos móveis com sistema operacional Android com versão superior a Jellybean 4.1, tanto de forma on-line quanto off-line. Assim, o aplicativo estará disponível em dispositivos de baixo custo e sem a necessidade de estar conectado a Internet.

As quatro principais ferramentas utilizadas para a construção do aplicativo foram: plataforma Unity 3D (mais detalhes em https://unity3d.com), Vuforia Engine (mais detalhes em https://library.vuforia.com), marcadores e smartphone com câmera. A Unity 3D foi utilizada para desenvolver o aplicativo como um todo, já que é uma plataforma que oferece aos desenvolvedores ferramentas para criação de softwares em 2D, 3D, realidade virtual e RA. Já o Vuforia Engine foi usado para trabalhar com RA, pois é um kit de desenvolvimento de software que pode ser usado para criar aplicativos de realidade aumentada integrado com a Unity 3D. Os marcadores de prismas e pirâmides foram feitos de acordo com a planificação do sólido a ser visualizado; já os do cilindro, cone e esfera foram feitos com base na formação do sólido por revolução.

Para atender os requisitos educacionais, buscou-se em planos de aula (http://portaldoprofessor.mec.gov.br) para o nível de Educação de Jovens e Adultos $1^{\circ}$ ciclo, Ensino Fundamental e Ensino Médio com os temas grandezas e medidas, geometria e espaço e forma. Dessa forma, a RA baseada em imagem foi utilizada para 
abordar conteúdos, como: prismas, pirâmides, cilindros, cones e esferas. Além disso, para que seja possível avaliar o conhecimento adquirido com o uso do aplicativo, foi proposta uma seção de perguntas de múltipla escolha sobre os sólidos geométricos. Estes foram os requisitos iniciais, os quais foram consolidados após a comparação com outros softwares semelhantes da área, o que será melhor detalhado na Tabela 1.

Considerando utilizar boas práticas em Engenharia de Software para elaborar o aplicativo, os requisitos foram consolidados através da utilização da técnica de análise de competidores como proposto pelo método de desenvolvimento em Vaupel et al. (2018). Assim, foram encontrados 3 aplicativos relacionados: GeoAR (Reis e Kirner, 2012), Siseuler (Lemos e Carvalho, 2010) e Geotransform3D (Barbosa e Carvalho, 2017). Deles, foram retirados alguns requisitos: a planificação dos sólidos, fórmulas de área e/ou volume, conter perguntas e animação de formação do sólido. O GeometriAR teve seu diferencial no foco do estudo e na animação de formação do sólido (Tabela 1), que traz a formação dos sólidos em 3D a partir de planos em duas dimensões, buscando auxiliar em uma construção do conhecimento mais significativa para os alunos.

Tabela 1 - Comparação de trabalhos relacionados.

\begin{tabular}{|c|c|c|c|c|}
\hline & GeoAR & Siseuler & Geotransform3D & GeometriAR \\
\hline Foco do estudo & $\begin{array}{c}\text { Figuras de } \\
\text { geometria } \\
\text { plana }\end{array}$ & $\begin{array}{l}\text { Sólidos que } \\
\text { podem ser } \\
\text { formados da } \\
\text { relação de Euler }\end{array}$ & $\begin{array}{c}\text { Prismas: } \\
\text { triangular, } \\
\text { quadrangular, } \\
\text { pentagonal e } \\
\text { hexagonal }\end{array}$ & $\begin{array}{c}\text { Prismas } \\
\text { (triangular e } \\
\text { quadrangular), } \\
\text { pirâmides, cone, } \\
\text { cilindro e esfera }\end{array}$ \\
\hline $\begin{array}{c}\text { Contém planificação } \\
\text { dos sólidos }\end{array}$ & Não & Não & Sim & Sim \\
\hline $\begin{array}{c}\text { Contém fórmulas de } \\
\text { área e/ou volume }\end{array}$ & Sim & Não & Não & Sim \\
\hline Contém perguntas & Sim & Não & Não & Sim \\
\hline \begin{tabular}{|c|} 
Contém animação de \\
formação do sólido
\end{tabular} & Não & Não & Não & Sim \\
\hline
\end{tabular}

Fonte: Os autores (2019).

Parte 2: A avaliação do aplicativo foi feita com base na opinião de usuários especialistas como proposto em Dias et al. (2013) e utilizado trabalho de Batista et al. (2018). Para isso, foram selecionados nove professores de matemática das cidades de Petrolina-PE, Juazeiro-BA e Sobradinho-BA. A quantidade de participantes se baseou nos resultados do trabalho de Nielsen (2000) que afirma que os melhores resultados de avaliação de usabilidade são obtidos através de testes com cinco usuários.

As questões feitas na avaliação foram baseadas na Ferramenta Pedagógica para Avaliação Ergonômica de Software Educativo (Pedagogical Ergonomic Tool for Educational Software Evaluation - PETESE), feita por Coomans e Lacerda (2015). A PETESE consiste em um guia de avaliação de softwares educacionais matemáticos.

A operacionalização foi dada de forma individual, assim, cada participante recebeu instruções para baixar o aplicativo e como usar os marcadores. Após utilizarem o aplicativo, eles responderam o questionário feito no Formulários Google, com base nas questões apresentadas no PETESE. As questões foram divididas em cinco aspectos: aspectos gerais, pedagógicos, de usabilidade, de conteúdo e de interface. A forma de resposta para cada questão foi disposta com a utilização da escala de Likert, para um melhor grau de conformidade, em que o usuário escolhe uma opção de resposta entre as 
cinco seguintes alternativas: concordo fortemente, concordo, nem concordo e nem discordo, discordo e discordo fortemente. No final do questionário foi colocado a opção de comentários adicionais, para possíveis sugestões ou observações. Após as avaliações realizadas, foram analisadas todas as respostas e feitas as discussões avaliativas.

\section{Resultados e discussões}

\subsection{O aplicativo GeometriAR}

O GeometriAR contém três seções principais: a visualização dos sólidos em RA, a parte de perguntas relacionadas ao assunto e a tela de ajuda com instruções de uso do aplicativo. Na Figura 1, pode ser visto o aplicativo funcionando no smartphone na tela de visualização de um sólido em 3D com base no marcador (figura impressa no papel).

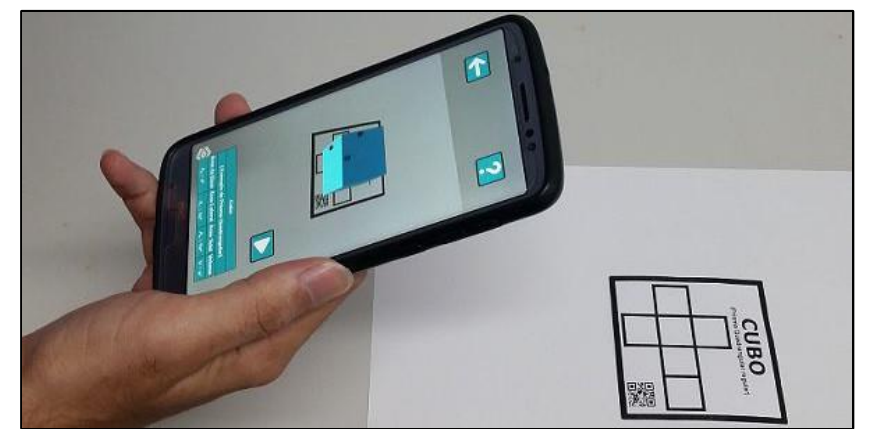

Figura 1 - Visualização de um cubo em 3D ao apontar para o marcador.

Como o aplicativo utiliza realidade aumentada baseada em imagem, foram feitos marcadores para representarem os seguintes sólidos geométricos: um prisma triangular, dois prismas quadrangulares, uma pirâmide triangular, uma pirâmide quadrangular, um cilindro, um cone e uma esfera. Portanto, para o funcionamento da visualização do sólido geométrico em realidade aumentada no aplicativo, é necessário que se aponte a câmera do dispositivo para um determinado marcador e assim será projetado um sólido em 3D em cima desse marcador, como mostrado na Figura 01.

$\mathrm{Na}$ tela de visualização do sólido, além do sólido projetado em 3D, também é mostrado seu nome e suas respectivas fórmulas de áreas e volume. Outro ponto que vale ser destacado, é que ao apertar o botão play (botão logo acima da tabela de fórmulas de cada sólido) é reproduzida uma animação de formação do sólido geométrico, para parar a animação é só apertar o botão pause (botão que substitui o botão play durante a animação). Um passo a passo da animação da formação de uma pirâmide quadrangular pode ser visto nas 3 sequências da Figura 2 .

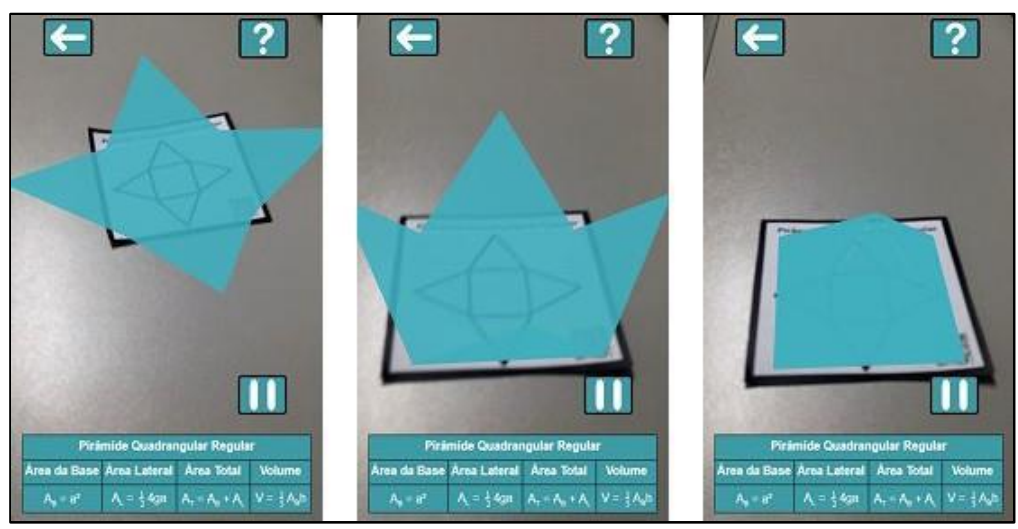

Figura 2 - Três frames da animação de formação de um sólido geométrico. 


\subsection{Avaliação do aplicativo}

Aspectos gerais: o aplicativo mostrou ser inovador, fácil de usar, condizente com o público-alvo e que pode ser usado em sala de aula. Como mostrado no Gráfico 1, houve uma discrepância entre as respostas com relação ao quesito de interdisciplinaridade, devido ao aplicativo ter um foco direcionado ao ramo de geometria espacial e não conter elementos que envolvem outras disciplinas. Já com relação aos diferentes níveis de complexidade, $22 \%$ dos avaliadores se mostraram indiferentes.

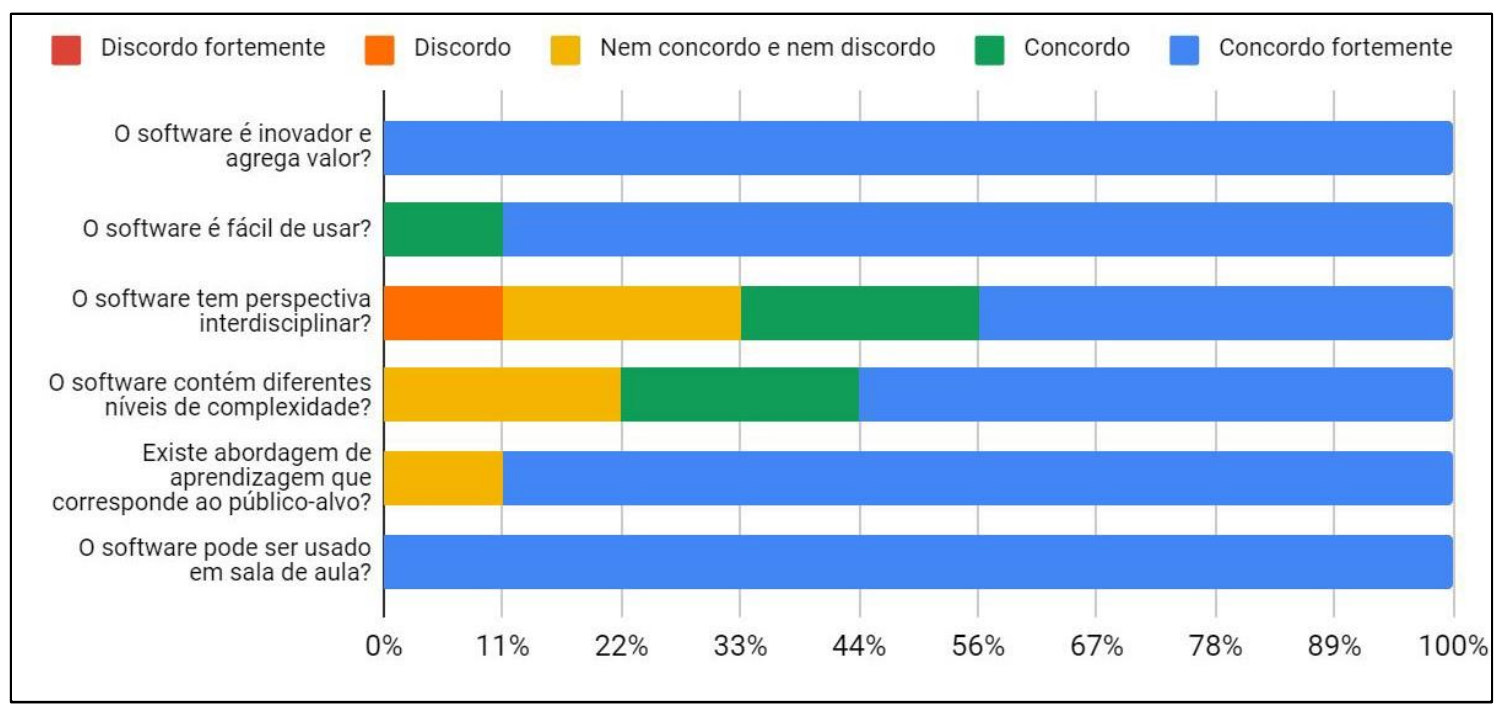

Gráfico 1 - Resultados da avaliação dos aspectos gerais.

Alguns fatores, que podem ter contribuído para esses resultados terem sido positivos, são: as necessidades de aulas diferentes, a não dependência de internet durante o uso do aplicativo e o crescimento do uso de smartphones. Assim, o resultado mostra, de acordo com o ponto de vista dos especialistas, a significância do aplicativo como um método inovador em sala de aula, a sua facilidade de uso e ainda que está alinhado ao público alvo. Destaque-se ainda que o aplicativo está alinhado com os resultados da pesquisa TIC Educação 2017 feita pelo Centro Regional de Estudos para o Desenvolvimento da Sociedade da Informação (2018), a qual mostra que o uso de celular em atividades escolares foi de $75 \%$ entre estudantes brasileiros do $2^{\circ}$ ano do ensino médio em 2017.

Aspectos pedagógicos: Os aspectos pedagógicos foram em sua maioria bem avaliados, como visto no Gráfico 2 mais adiante, mostrando que o aplicativo tem objetivos educacionais bem definidos, elementos de cooperação, de auto-análise, avaliação, tarefas e nível de dificuldade adequado ao público-alvo. As atividades trabalham os objetivos matemáticos propostos, isso se dá, provavelmente, por causa da possível interação e visualização do aluno com os sólidos geométricos de uma forma mais atrativa pelo uso da RA.

Portanto, os especialistas apontam que o aplicativo é possível de ser utilizado como ferramenta pedagógica para o caso de sólidos geométricos. O resultado se mostra semelhante com outras tecnologias que usam a perspectiva construtivista para o ensino de sólidos geométricos (Kynigos, 2015). Destaca-se, que o papel dos professores continua sendo preciso para a construção do conhecimento do aluno, sendo eles tutores para a utilização do software de forma adequada. 


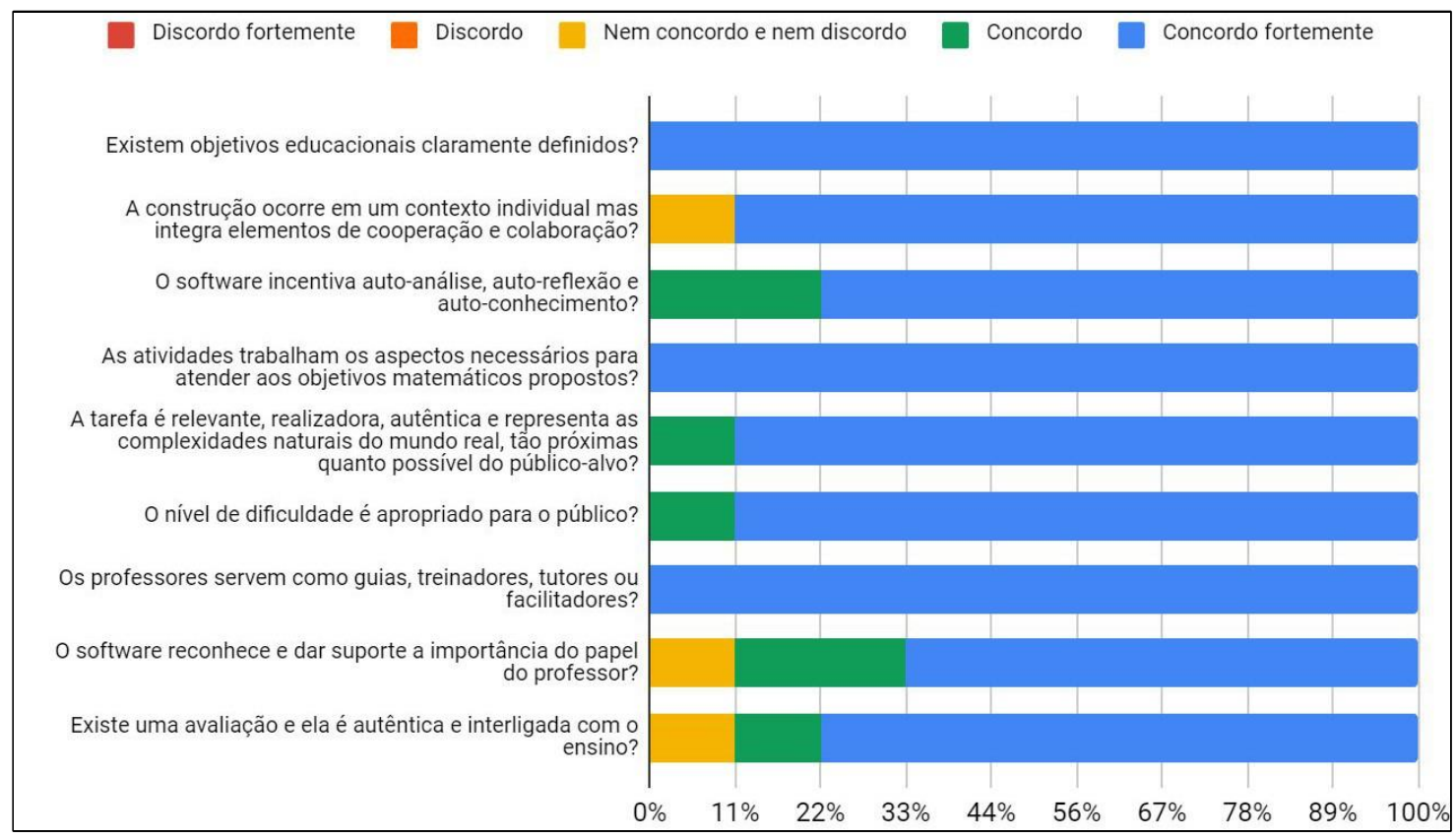

Gráfico 2 - Resultados da avaliação dos aspectos pedagógicos

Usabilidade: No Gráfico 3 são mostradas as avaliações de usabilidade. O recurso de ajuda, as instruções de uso, as interações com o sistema e o controle sobre o sistema tiveram uma boa avaliação. Assim, o resultado da avaliação da usabilidade mostrou que o aplicativo é possível de ser usado, entretanto, a avaliação do recurso de feedback, apesar de ser empregado, pode não ter sido unânime nas avaliações, devido a, especificamente, na parte das perguntas do aplicativo não serem detalhadas as soluções corretas das perguntas que foram respondidas erradas, e/ou não ter um recurso a mais para auxiliar a busca pelas as respostas corretas.

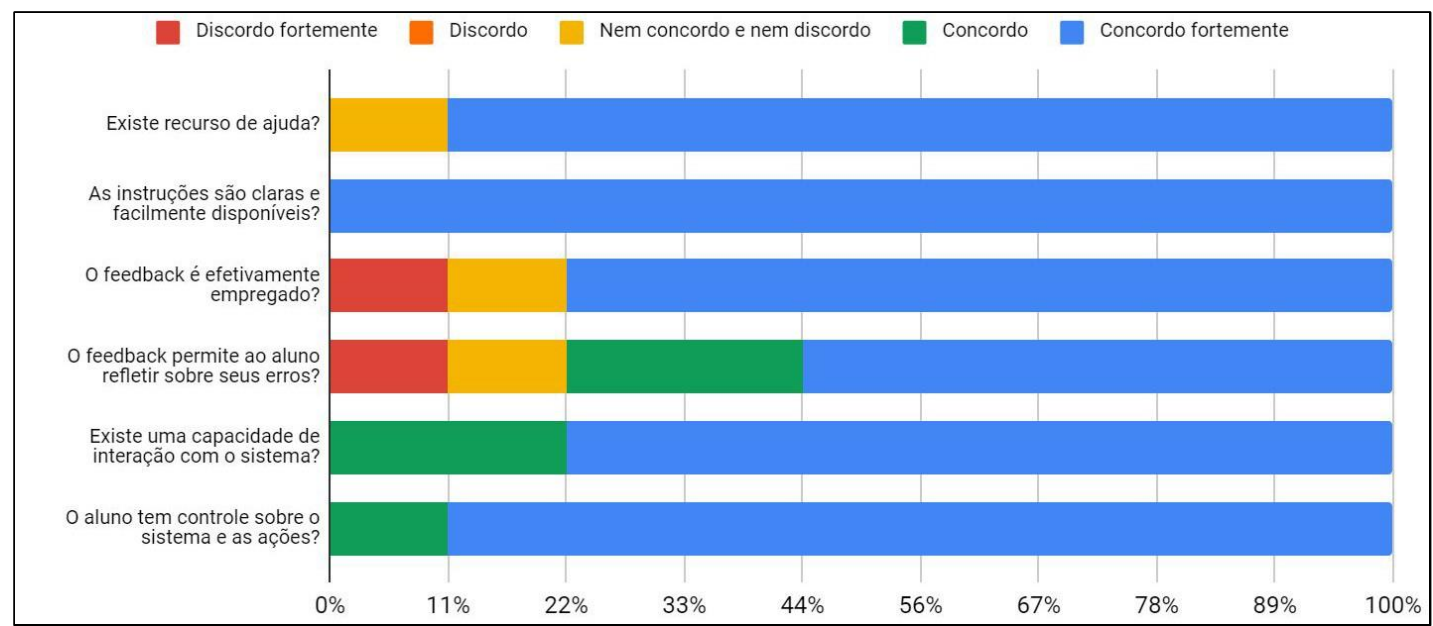

Gráfico 3 - Resultados da avaliação de usabilidade.

Conteúdo do aplicativo: são compostos pelos termos matemáticos, animação de formação dos sólidos geométricos e as imagens em 2D e 3D contidas no aplicativo. Eles também obtiveram boas avaliações, como representado no Gráfico 4. A linguagem e os termos matemáticos se apresentaram adequados ao público-alvo, o conteúdo se mostra claro e compreensível, e os elementos multimídia são apropriados e auxiliam na compreensão dos sólidos geométricos. Esse resultado foi tido como um bom indicativo de que o aplicativo está com o seu conteúdo de acordo com o proposto. 


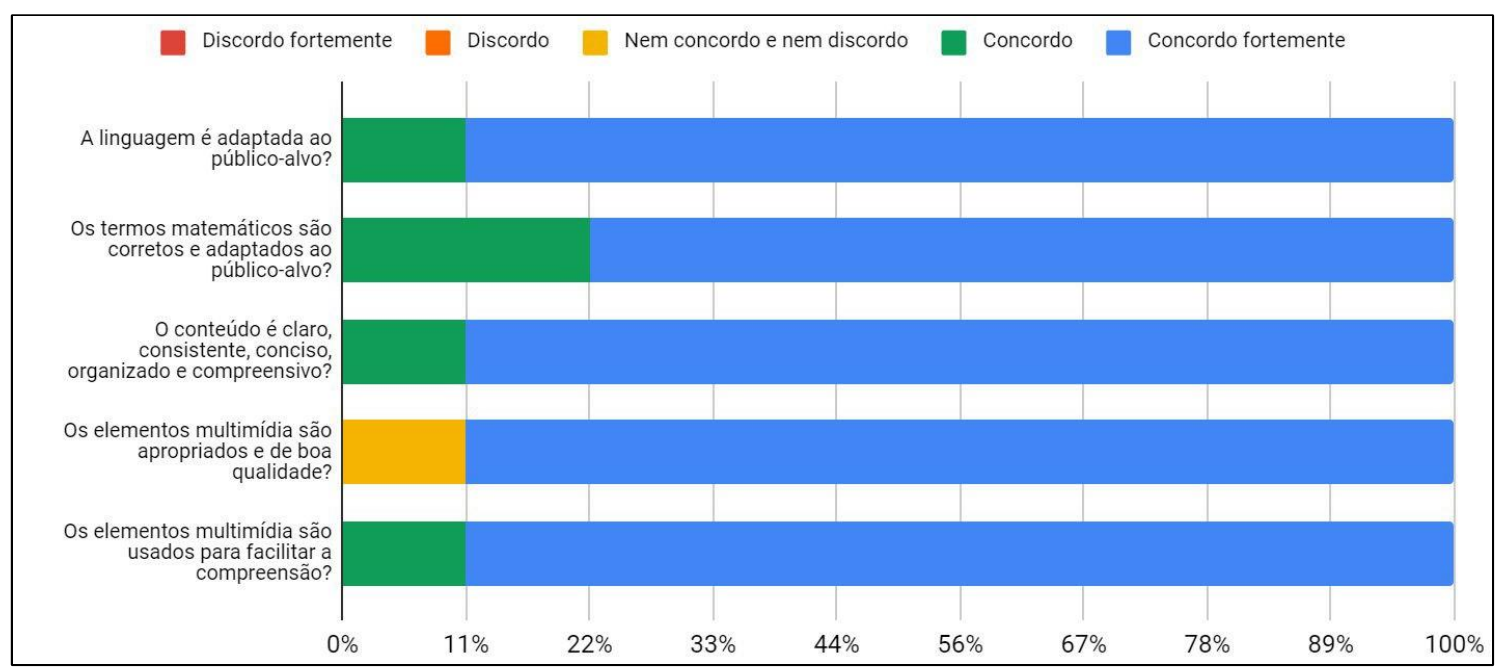

Gráfico 4 - Resultados da avaliação de conteúdo.

Interface: No Gráfico 5, tem-se a avaliação da interface, ela demonstra que a estrutura do aplicativo é clara e compreensível, a tipografia é apropriada, o design é agradável e os ícones são representativos. As cores, para $22 \%$ dos avaliadores, foram indiferentes quanto a sua significação e ajuda na compreensão do software. A usabilidade e a interface são fatores que influenciam no conceito de experiência do usuário (Preece et al., 2015). Por isso, é importante uma boa avaliação nesses quesitos, para que os usuários tenham uma boa experiência no uso do aplicativo e não tenham frustrações.

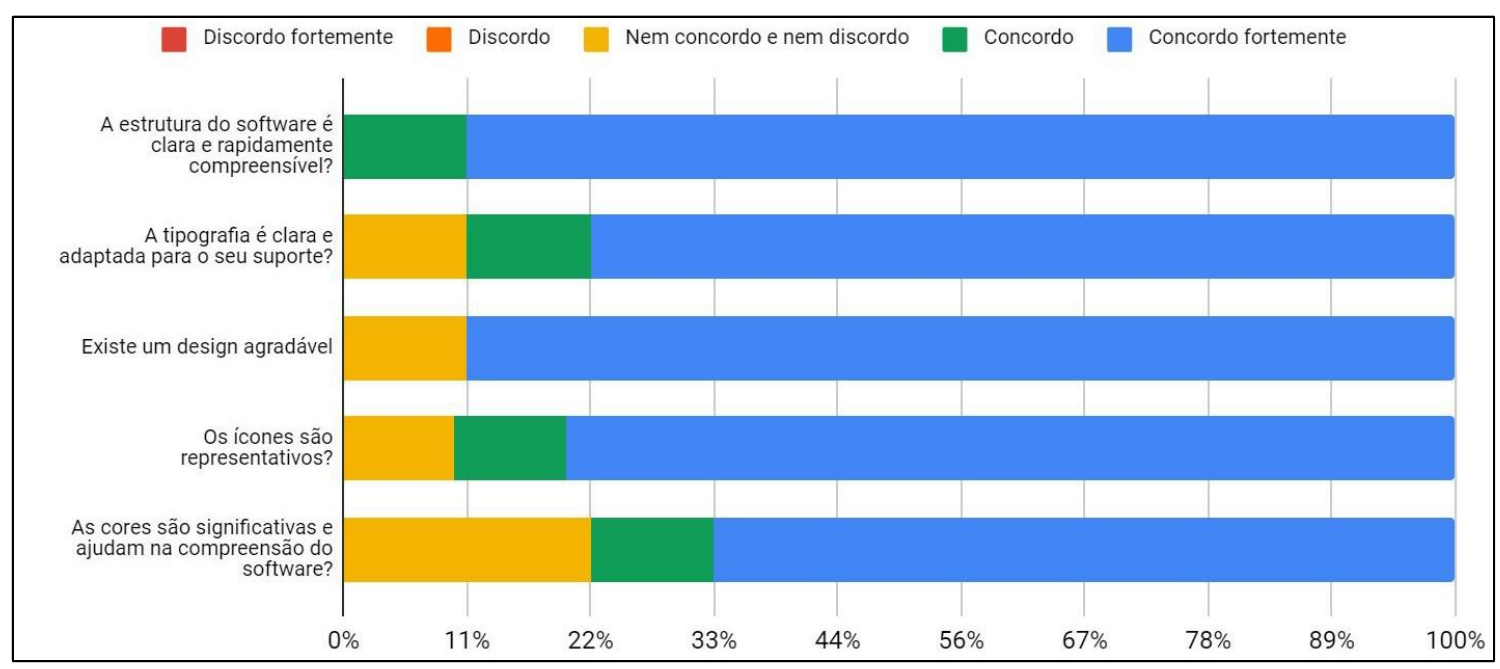

Gráfico 5 - Resultados da avaliação de interface.

Comentários adicionais: Em relação às respostas da questão sobre comentários adicionais, a maioria dos professores elogiou o aplicativo e alguns já estão o utilizando em sala de aula, dois professores fizeram observações referentes às questões do aplicativo, para que elas fossem mais contextualizadas e relacionadas com as do ENEM (Exame Nacional do Ensino Médio), com isso o aplicativo teria maior interdisciplinaridade. Um outro professor fez uma sugestão de colocar mais sólidos geométricos. Esses resultados serão importantes para a próxima versão do aplicativo. 


\section{Considerações finais}

Este artigo foca em sugerir uma solução para ser utilizada por professores no ensino e na compreensão dos sólidos geométricos através da sua visualização em três dimensões. Assim, foi desenvolvido o GeometriAR, um aplicativo que faz o uso de RA e se apresenta como uma proposta para auxiliar no ensino de sólidos geométricos através da percepção do aluno na construção do sólido em 3D.

Com base nas avaliações feitas pelos professores de matemática, o aplicativo se mostrou relevante para ser utilizado em sala de aula no ensino de geometria espacial devido a sua qualidade pedagógica, sua usabilidade, seu conteúdo, sua interface e seus aspectos gerais. Novas melhorias ao aplicativo serão constantes, a exemplo de uma versão com a adição de novos sólidos geométricos e alterações nas questões da parte de perguntas do aplicativo. Isso será possível, pois o aplicativo se encontra disponível gratuitamente para qualquer usuário faça o download e ainda para que os usuários deem sua opinião sobre o aplicativo.

Como trabalho futuro é possível a realização de projetos de avaliação em maior escala do GeometriAR, e avaliação por meio dos estudantes com um estudo caso controle para aferir o ganho de aprendizagem e/ou atenção à medida que eles utilizem o aplicativo. Desse modo, haja vista a dinâmica do mundo contemporâneo cujo principal destaque cada vez mais é a tecnológica, este artigo e seus desdobramentos têm sua relevância social ao buscar contribuir para um ensino atualizado, digital e significativo.

\section{REFERÊNCIAS}

AKÇAYIR, M.; AKÇAYIR, G. Advantages and challenges associated with augmented reality for education: a systematic review of the literature. Educational Research Review, v. 20, p. 1-11, 2017.

AZUMA, R. T. A survey of augmented reality. Presence: Teleoperators \& Virtual Environments, v. 6, n. 4, p. 355-385, 1997.

BARBOSA, J. W. S.; CARVALHO, C. V. de A. GEOTRANSFORM3D: objeto computacional em realidade aumentada para apoio ao ensino da matemática. Revista de Educação, Ciências e Matemática, v. 7, n. 1, 2017.

BATISTA, M. F.; RAMOS, R. A.; DE BRITO, L. F. Utilizando o Aplicativo Criptomática para Ensinar Conteúdos Matemáticos do Ensino Médio com Uso da Criptografia. RENOTE, v. 16, n. 2, 2018.

CADAVIECO, A. J. F.; GOULAO, M. de F.; TAMARGO, M. A. G. Melhorar a atratividade da informação através do uso da realidade aumentada. Perspectivas em Ciência da Informação, 2014.

CENTRO REGIONAL DE ESTUDOS PARA O DESENVOLVIMENTO DA SOCIEDADE DA INFORMAÇÃO. TIC educação 2017: pesquisa sobre o uso das tecnologias de informação e comunicação nas escolas brasileiras. São Paulo: Comitê Gestor da Internet no Brasil, 2018.

CHAVES, J. de O. Geometria espacial no ensino fundamental: uma reflexão sobre as propostas metodológicas. Viçosa: UFV, 2013. 78p. Dissertação de Mestrado. CHENG, Kun-Hung; TSAI, Chin-Chung. Affordances of augmented reality in science learning: suggestions for future research. Journal of Science Education and Technology, v. 22, n. 4, p. 449-462, 2013.

COOMANS, S.; LACERDA, G. S. Petese: a pedagogical ergonomic tool for educational software evaluation. Procedia Manufacturing, v. 3, p. 5881-5888, 2015. DIAS, J.; NASCIMENTO, F. S.; HETKOWSKI, T. M.; BRANDÃO, I.; PEREIRA, T. R. D. S. Avaliação de jogos educacionais digitais baseada em Perspectivas: Uma 
experiência através do Jogo-simulador Kimera. In: Anais do Simpósio Brasileiro de Jogos e Entretenimento Digital. 2013. v. 1, p. 1, 2013.

HERPICH, F.; BOS, A.; KUHN, I.; GUARESE, R. L. M.; TAROUCO, L. M. R.; WIVES, L.; ZARO, M. A. Atividade cerebral no uso de recursos educacionais em realidade aumentada: uma análise da atenção do aprendiz. In: Simpósio Brasileiro de Informática na Educação (SBIE). 2018. p. 1858-1862.

INSTITUTO NACIONAL DE ESTUDOS E PESQUISAS EDUCACIONAIS ANÍSIO TEIXEIRA. Brasil no PISA 2015: análises e reflexões sobre o desempenho dos estudantes brasileiros. Brasília, 2016.

INSTITUTO NACIONAL DE ESTUDOS E PESQUISAS EDUCACIONAIS ANÍSIO TEIXEIRA. Press kit SAEB 2017. Brasília, 2018.

KYNIGOS, C. Constructionism: Theory of Learning or Theory of Design?. In: Selected regular lectures from the 12th International Congress on Mathematical Education. Springer, Cham, 2015. p. 417-438.

LEMOS, B. M.; CARVALHO, C. V. de A. Uso de realidade aumentada para apoio ao entendimento da relação de Euler. RENOTE, v. 8, n. 2, 2010.

MARQUES, V. D.; CALDEIRA, C. da C. Dificuldades e carências na aprendizagem da Matemática do Ensino Fundamental e suas implicações no conhecimento da Geometria. Revista Thema, v. 15, n. 2, p. 403-413, 2018.

MASMUZIDIN, M. Z.; AZIZ, N. The Current Trends of Augmented Reality in

Early Childhood Education. The International Journal of Multimedia \& Its

Application, v. 10, n. 6, p. 47-58, 2018.

MEKNI, M.; LEMIEUX, A. Augmented reality: applications, challenges and future trends. Applied Computational Science, p. 205-214, 2014.

MENEGAI, D. A. F. N.; D’AVILA, J. A.; FAGUNDES, D. da S.; FERREIRA, V. L. D. Formação continuada: Integração das Tecnologias Digitais na Prática Pedagógica de Professores de Matemática. RENOTE, v. 16, n. 2, 2018.

MILGRAM, P.; TAKEMURA, H.; UTSUMI, A.; KISHINO, F. Augmented reality: a class of displays on the reality-virtuality continuum. In: Telemanipulator and telepresence technologies. International Society for Optics and Photonics, 1995.

NIELSEN, J. Why you only need to test with 5 users. 2000. Disponível em: <https://www.nngroup.com/articles/why-you-only-need-to-test-with-5-users/>. Acesso em: 20 maio 2019.

PREECE, J.; ROGERS, Y.; SHARP, H. Interaction design: beyond human-computer interaction. John Wiley \& Sons, 2015.

REIS, F. M. V.; KIRNER, T. G. Percepção de estudantes quanto à usabilidade de um livro interativo com realidade aumentada para a aprendizagem de geometria. RENOTE, v. 10, n. 1, 2012.

SALIN, E. B. Geometria espacial: a aprendizagem através da construção de sólidos geométricos e da resolução de problemas. Revemat: Revista Eletrônica de Educação Matemática, v. 8, n. 2, p. 261-274, 2013.

VIDALETTI, V. B. B. Ensino e aprendizagem da geometria espacial a partir da manipulação de sólidos. Lageado: UNIVATES, 2009. p. 15. Dissertação de Mestrado. VAUPEL, S.; GERLACH, R.; GUCKERT, M. Model-driven development of mobile applications for Android and iOS supporting role-based app variability. Software \& Systems Modeling, v. 17, n. 1, p. 35-63, 2018.

VOLPATTO, A. T.; FORTES, P. R.; SILVEIRA, S. R. Um estudo de caso envolvendo a aplicação de um software educacional de geometria espacial. Revemat: Revista Eletrônica de Educação Matemática, v. 13, n. 1, p. 76-90, 2018. 